\title{
Two-Sided Controlled Transition in Biorthogonal Time-Varying Filter Banks
}

\author{
Aweke N. Lemma and Ed F. Deprettere
}

\begin{abstract}
Using state space representations of biorthogonal time-varying filter banks, it is possible to derive conditions under which the transition between two time-invariant filter banks can be so controlled that both the decomposition filters and the reconstruction functions gently embrace. The transition interval can be made arbitrarily short. If it is zero, then the special case of instantaneous transition is reached.
\end{abstract}

\section{INTRODUCTION}

$\mathbf{T}$ THE subject of filter banks has received an increasing attention in the recent decade. This is because they appear in various forms and for various reasons in multimedia and wireless communication applications. Particularly, people have started putting considerable efforts in the time-varying aspect of filter banks. To come up with a profound theory, most researchers concentrate on the study of transitions between two stationary filter banks. The reason for such considerations is partly that most of the time, filters tend to operate for considerable duration compared with their lengths and can be assumed to be stationary at the time of transition.

Generally, when changing from one stationary filter to another, we may have one of the following conditions: either i) transition is required, or ii) transition is not required. The first case is studied by a number of authors [1]-[4]. The first three papers consider an equal number of transition filters both on the decomposition and reconstruction sides, whereas in [4], unequal transition segments are studied. The filter banks we consider here do not lie under this first category.

The second case-switching between two stationary filters without any transition-is considered in [5]. There, it is shown that if the filters under question are related in a particular way, instantaneous transition both on the decomposition and reconstruction sides is possible. This not withstanding, it might make sense from other considerations to go smoothly, and instead of going back to case i), we propose that we make a controlled transition without requiring any additional optimization procedure. The behavior in the transition period is controlled by careful choice of the trajectory on which the filters transit.

Manuscript received May 9, 1995. This work was supported by Philips Research, The Netherlands. The associate editor coordinating the review of this letter and approving it for publication was Prof. P. P. Vaidyanathan.

The authors are with the Department of Electrical Engineering, Delft University of Technology, Delft, The Netherlands.

Publisher Item Identifier S 1070-9908(96)01833-0.

\section{Biorthogonal Filter Banks}

A biorthogonal filter bank $(E, R)$ is a special form of linear expansion of a sequence or signal. Thus if $u \in l_{2}^{P}(\mathbb{Z}),{ }^{1}$ then $u=\sum\left\langle u, e_{:, n}\right\rangle r_{n,:}$, where $e_{:, n}$ and $r_{n,:}$ are the columns and rows of bounded matrix operators $E$ and $R$, respectively, with $E R=I$. We assume here that the entries of these operators are finite dimensional matrices of dimension $P \times Q$ and $Q \times P$, respectively, with $Q \geq P$. If $Q=P$, then $R E=I$ as well. If the filter bank is shift invariant, then $E$ and $R$ are Toeplitz and characterized by the central column $e$ and central row $r$, respectively. In this case, we write $(E(e), R(r))$ instead of $(E, R)$.

$$
\begin{aligned}
& E(e) \\
& =\left[\begin{array}{ccccccc}
\ddots & \vdots & \vdots & \vdots & \vdots & \vdots & \\
& e(0) & e(1) & \ldots & \ldots & e(k+1) & \ldots \\
& & \mathrm{e}(0) & e(1) & \ldots & e(k) & \ldots \\
& & & e(0) & \ldots & e(k-1) & \ldots \\
& & & & \ddots & \vdots & \vdots \\
& & & & & e(0) & \ldots \\
& & & & & & \ddots
\end{array}\right]
\end{aligned}
$$

$$
\begin{aligned}
& R(r) \\
& =\left[\begin{array}{ccccccc}
\ddots & & & & & & \\
\ldots & r(0) & & & & & \\
\vdots & \vdots & \ddots & & & & \\
\ldots & r(k-1) & \ldots & r(0) & & & \\
\ldots & r(k) & \ldots & r(1) & \mathrm{r}(0) & & \\
\ldots & r(k+1) & \ldots & \ldots & r(1) & r(0) & \\
& \vdots & \vdots & \vdots & \vdots & \vdots & \ddots
\end{array}\right] .
\end{aligned}
$$

In the context of filter banks, the columns of $E$ are called filter weight vectors, and the rows of $R$ are called impulse response vectors. Moreover, they have the property that with $F(x)$, the Fourier transform of $x, F(e)$ and $F(r)$ are uniform spectral decompositions of the base band $[0, \pi] .^{2}$

In most applications a single biorthogonal filter bank $(E(e), R(r))$ is used to decompose and reconstruct certain

\footnotetext{
${ }^{1}$ That is, $u$ is a finite energy sequence or row vector with $P$-dimensional row vector entries $u(n), n \in \mathbb{Z}$.

${ }^{2} F(e)$ and $F(r)$, respectively, correspond to the $\mathbf{h}^{T}(z)$ and $\mathbf{f}(z)$ given in page 224 of [6].
} 


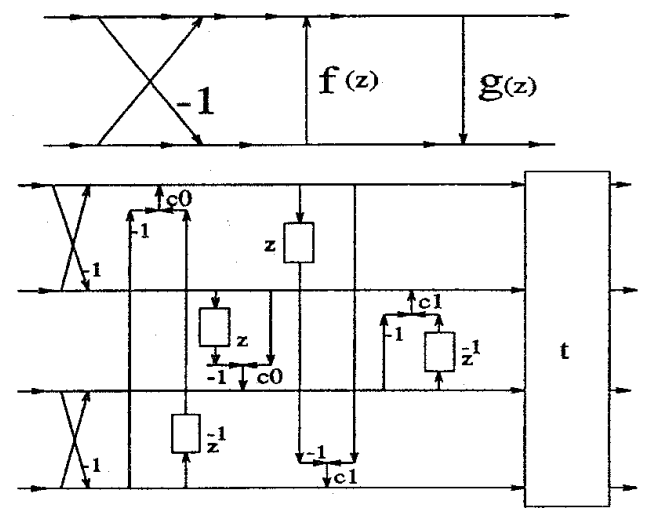

Fig. 1. Decomposition parts of two- and four-channel perfect reconstruction filter banks.

signals $u$. However, as most signals are not stationary, it may be expected that time-varying filter banks will do a better job, especially when a quantization operator is inserted in between the two bank operators. In the latter case, for example, it is then also necessary to guarantee that the spectral decomposition properties of the time-varying filter weight vectors are preserved and that the time-varying impulse response vectors remain good interpolation functions.

In this letter, we find perfect reconstruction filter banks giving signal expansions of the form

$$
\begin{aligned}
u= & \sum_{n=-\infty}^{n_{0}-1}\left\langle u, e 1_{:, n}\right\rangle r 1_{n,:} \\
& +\sum_{n=n_{0}}^{n_{f}-1}\left\langle u, e_{:, n}\right\rangle r_{n,:}+\sum_{n=n_{f}}^{+\infty}\left\langle u, e 2_{:, n}\right\rangle r 2_{n,:}
\end{aligned}
$$

where $n_{f} \geq n_{0}$, the $e 1_{:, n}$, and the $e 2:, n$ are all shifted versions of $e 1$ and $e 2$, respectively (and similarly for the $r 1_{n,:}$ and the $r 2_{n,:}$ ). In other words, the behavior on the segments $\left[-\infty, n_{0}-\right.$ 1] and $\left[n_{f},+\infty\right]$ is shift invariant, whereas the segment $\left[n_{0}, n_{f}-1\right]$ is the transition region. In the next section, we summarize results for the case $n_{0}=n_{f}$, which were presented in [5] and allow the construction of piecewise stationary biorthogonal banks. In Section III, we extend these results to the case when $n_{f}>n_{0}$ by introducing an interpolation method that allows smooth transition between filter weight vectors in the decomposition part of the bank and, at the same time, smooth transition between impulse response vectors in the reconstruction part of the bank. Examples that demonstrate the results are given in Section IV.

\section{InSTANTANEOUS TRANSITION IN PERFECT RECONSTRUCTION FILTER BANKS}

Let $(E(e), R(r))$ be a shift-invariant biorthogonal filter bank. Put $E(e)=\left[E_{l} \mid E_{r}\right]$ and $R(r)=\left[\frac{R_{t}}{R_{b}}\right]$, where $E_{l}$ is the part of $E(e)$ to the left of the central column $e$, and $R_{t}$ is the part of $R(r)$ above the central row $r$.

Now, given $E_{1}\left(e_{1}\right)=\left[E_{1, l} \mid E_{1, r}\right], E_{2}\left(e_{2}\right)=\left[E_{2, l} \mid E_{2, r}\right]$ and $R_{1}\left(r_{1}\right)=\left[\frac{R_{1, t}}{R_{1, b}}\right], R_{2}\left(r_{2}\right)=\left[\frac{R_{2, t}}{R_{2, b}}\right] . E_{1}\left(e_{1}\right) R_{1}\left(r_{1}\right)=I$ and $E_{2}\left(e_{2}\right) R_{2}\left(r_{2}\right)=I$, we address the following problem.
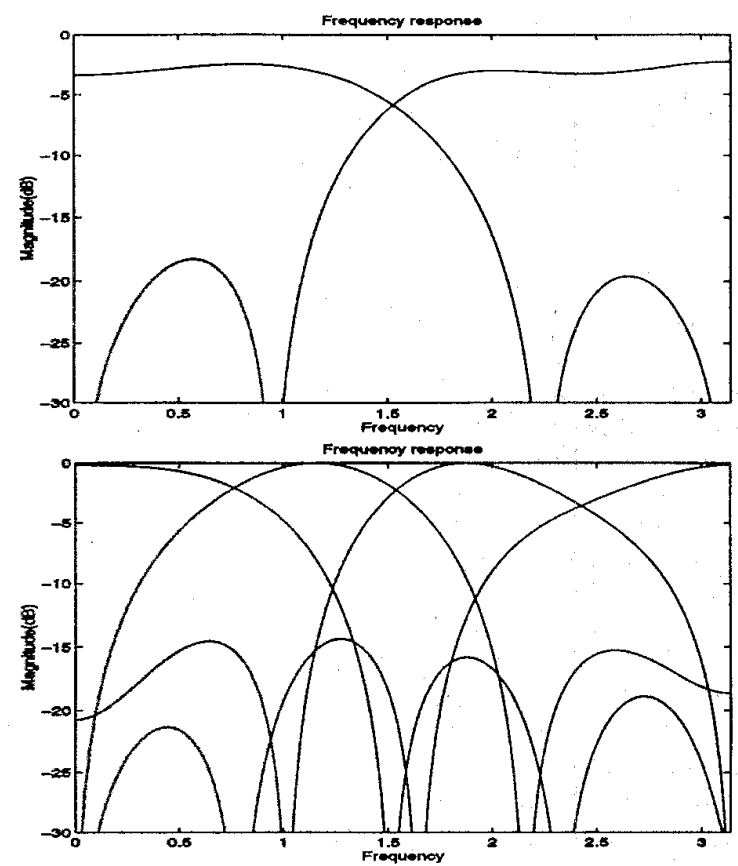

Fig. 2. Fourier transforms of filter weight vectors of the two- and four-channel filters.

Problem: Given the above two filter banks $\left(E_{1}\left(e_{1}\right)\right.$, $\left.R_{1}\left(r_{1}\right)\right)$ and $\left(E_{1}\left(e_{1}\right), R_{1}\left(r_{1}\right)\right)$, does there exist a filter bank $\left(E_{12}, R_{12}\right)$ such that $E_{12}=\left[E_{1, l} \mid E_{2, r}\right]$ and $R_{12}=\left[\frac{R_{1, t}}{R_{2, b}}\right]$ (and of course $E_{12} R_{12}=I$ )?

The answer is partly contained in the following proposition, a proof of which can be found in [5].

Proposition 1: Let $\left(E_{1}\left(e_{1}\right), R_{1}\left(r_{1}\right)\right)$ and $\left(E_{2}\left(e_{2}\right), R_{2}\left(r_{2}\right)\right)$ be two shift-invariant biorthogonal filter banks with realization matrix pairs:

$$
\left(m_{1}\left(a_{1}, b_{1}, c_{1}, d_{1}\right), \bar{m}_{1}\left(\bar{a}_{1}, \bar{b}_{1}, \bar{c}_{1}, \bar{d}_{1}\right)\right)
$$

and

$$
\left(m_{2}\left(a_{2}, b_{2}, c_{2}, d_{2}\right), \bar{m}_{2}\left(\bar{a}_{2}, \bar{b}_{2}, \bar{c}_{2}, \bar{d}_{2}\right)\right)
$$

respectively. If ${ }^{3} a_{1}=a=a_{2}, b_{1} b=b_{2}, \bar{a}_{1}=\bar{a}=\bar{a}_{2}$, $\bar{c}_{1}=\bar{c}=\bar{c}_{2}, c_{2}=c_{1} \times t, d_{2}=d_{1} \times t, \bar{b}_{2}=s \times \bar{b}_{1}$, $\bar{d}_{2}=s \times \bar{d}_{1}$, and $t \times s=I$

$$
\begin{gathered}
I=\left[\begin{array}{ll}
a & c_{1} \\
b & d_{1}
\end{array}\right] \cdot\left[\begin{array}{cc}
\bar{a} & \bar{c} \\
\bar{b}_{1} & \bar{d}_{1}
\end{array}\right]= \\
{\left[\begin{array}{cc}
a & c_{1} \\
b & d_{1}
\end{array}\right] \cdot\left[\begin{array}{ll}
I & 0 \\
0 & t
\end{array}\right] \cdot\left[\begin{array}{ll}
I & 0 \\
0 & s
\end{array}\right] \cdot\left[\begin{array}{cc}
\bar{a} & \bar{c} \\
\bar{b}_{1} & \bar{d}_{1}
\end{array}\right]=} \\
{\left[\begin{array}{ll}
a & c_{2} \\
b & d_{2}
\end{array}\right] \cdot\left[\begin{array}{cc}
\bar{a} & \bar{c} \\
\bar{b}_{2} & \bar{d}_{2}
\end{array}\right]}
\end{gathered}
$$

then $\left(\left[E_{1, l} \mid E_{2, r}\right],\left[\frac{R_{1, t}}{R_{2, b}}\right]\right)$ is a biorthogonal filter bank with instantaneous filter weight vector transition in the decomposition part and instantaneous impulse response transition in the reconstruction part.

\footnotetext{
${ }^{3} \mathrm{Up}$ to similarity transformations.
} 


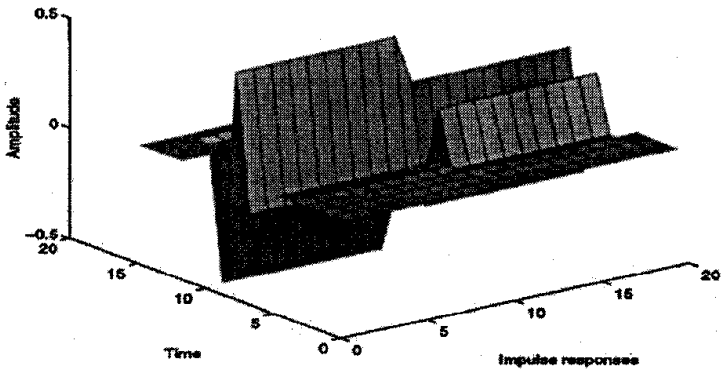

Fig. 3. Instantaneous switching in the impulse response corresponding to channel four.

In the above proposition, we have tacitly assumed that the two banks $\left(E_{1}\left(e_{1}\right), R_{1}\left(r_{1}\right)\right)$ and $\left(E_{2}\left(e_{2}\right), R_{2}\left(r_{2}\right)\right)$ have the same number of channels. This is, however, not a restriction. Indeed, if $m_{1}\left(a_{1}, b_{1}, c_{1}, d_{1}\right)$ is the realization matrix of - say-a p-channel filter, then a number of such realizations-say $r$-in (time) succession can be merged (by eliminating intermediate states) to obtain a realization matrix $m_{1}\left(a_{1}, b_{1}, c_{1}, d_{1}\right)$ of an $r \times p$ channel filter. The realization matrix $m_{2}\left(a_{2}, b_{2}, c_{2}, d_{2}\right)$ in the proposition will then also characterize an $r \times p$ filter bank. See the example in Section IV.

In the next section, we start from this result to extend the transition behavior from instantaneous to graceful.

\section{INTERPOLATED TRANSITION IN PERFECT RECONSTRUCTION FILTER BANKS}

The matrix equation in Proposition 1 expresses perfect reconstruction. The additional property is that the system is state stationary over all time including the time instant of instantaneous transition. The transition is instantaneous because the transformation matrices $t$ and $s$ are constant. If, on the other hand, we let these matrices be time varying, then the transition will follow a certain trajectory, which we will have to control for the case in which we want to enforce meaning on the trajectories of the filter weight vectors in the operator $E$ as well as the impulse response vectors in the operator $R$. One can envisage several strategies to control the transition behavior, but we will confine ourselves to one of them-spiral interpolation-that has proven to be simple and satisfactory.

Thus, let $\{t(n)\}$ be the sequence of real $w \times w$ transformation matrices on the transition interval $\left[t_{0}, t_{f}\right]$, where $t\left(n_{0}\right)=I$ and $t\left(n_{f}\right)=t, t$ being-for example-the matrix $t$ in Proposition 1. Associated with this sequence is the sequence of inverse matrices $\{s(n)\}, t(n) s(n)=I$. Now, let $t=g \lambda g^{-1}$ and $s=g \lambda^{-1} g^{-1}$ be the eigenvalue decompositions of $t$ and $s$. The eigenvalues are either real $\lambda_{i}$ or appear in conjugate pairs $\left(\left|\lambda_{k}\right| e^{j \theta_{k}},\left|\lambda_{k}\right| e^{-j \theta_{k}}\right)$.

Proposition 2: For $i=1, \ldots, w$ and $n_{0} \leq n \leq n_{f}$, let $p_{i}(n)$ and $\gamma_{i}(n)$ be real and monotonically increasing functions from 0 at $n_{0}$ to 1 at $n_{f}$. Put $t(n)=g\left[\operatorname{diag}\left(p_{i}(n)\left|\lambda_{i}\right|+(1-\right.\right.$ $\left.\left.\left.p_{i}(n)\right)\right) e^{j \gamma_{i}(n) \theta_{i}}\right] g^{-1} \cdot t(n)$ is real.

If $q_{i}(n)=\frac{p_{i}(n)\left|\lambda_{i}\right|}{p_{i}(n)\left|\lambda_{i}\right|+\left(1-p_{i}(n)\right)}$, then $q_{i}(n)$ is monotonically increasing from 0 at $n_{0}$ to 1 at $n_{f} ; s(n)=g\left[\operatorname{diag}\left(q_{i}(n)\left|\lambda_{i}^{-1}\right|+\right.\right.$ $\left.\left.\left(1-q_{i}(n)\right)\right) e^{j-\gamma_{i}(n) \theta_{i}}\right] g^{-1}$ is real, and $t(n) \times s(n)=I$.

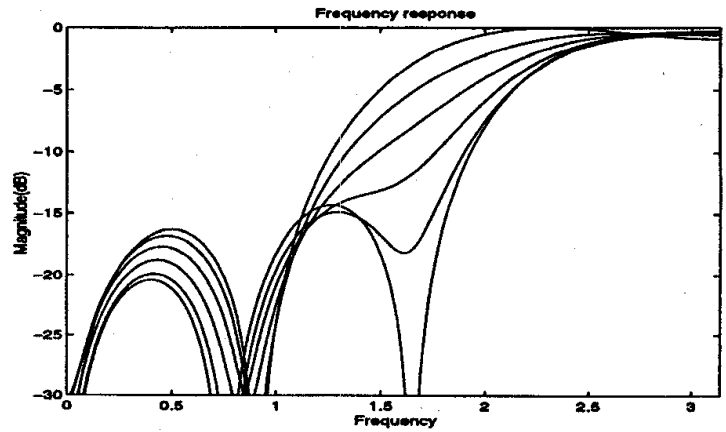

Fig. 4. Smooth transition in the frequency spectra corresponding to the fourth channel.

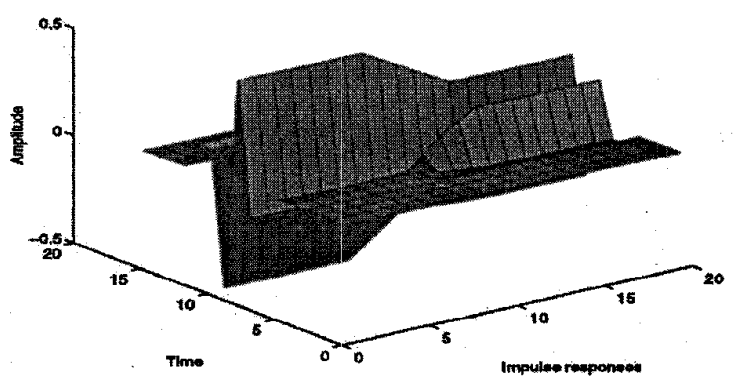

Fig. 5. Smooth transition in the impulse responses corresponding to the fourth channel.

\section{ILLUSTRATTVE EXAMPLES}

We consider, as an example, the case when a four-channel bank takes over from a two-channel bank. The decomposition parts of the two banks are shown in Fig. 1 in shift-invariant state. In this figure, $f(z)=-c_{0} . z+c_{0} \cdot z^{-1}$, and $g(z)=$ $-c_{1} . z+c_{1} . z^{-1} . t$ is a constant $4 \times 4$ matrix. The Fourier transforms of the filter weight vectors of the two decomposition parts are shown in Fig. 2.

\section{Instantaneous Transition}

If in the second filter in Fig. 1 the matrix $t$ is replaced by the identity, then the resulting flow graph is essentially twice the first filter. Thus, if $t$ is taken away from the second filter, then it represents two time steps of the two-channel decomposition filter. This filter can be run for a while and then-say at $n=n_{0}-t$ can be cascaded to the two two-channel filters and start running as a single four-channel decomposition filter. The takeover is instantaneous. That is, the spectral characteristics switch instantly, at $n=n_{0}$, from the top spectra in Fig. 2 to the bottom spectra. Moreover, the impulse responses of the reconstruction filters also have an instantaneous transition at $n=n_{0}$, as shown in Fig. 3 for the fourth channel of the two reconstruction filters. ${ }^{4}$

\footnotetext{
${ }^{4}$ The reconstruction filters are not shown as they are easily obtained by reversing the direction of signal flow from output to input in the filters from Fig. 1 .
} 


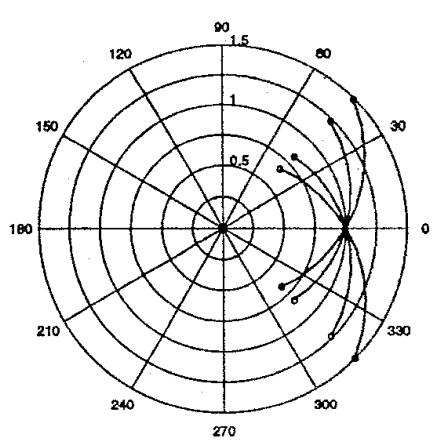

Fig. 6. Eigenvalue trajectories on the transition support. Initial values are all 1; end values are outside the unit circle for the decomposition filter and inside the unit circle for the reconstruction filter.

\section{Interpolated Transition}

Instead of appending the constant matrix $t$ at time instant $n_{0}$, we now "spiral," as explained in Proposition 2, along the matrix trajectory $t(n)=g\left[\operatorname{diag}\left(p_{i}(n)\left|\lambda_{i}\right|+(1-\right.\right.$ $\left.\left.\left.p_{i}(n)\right)\right) e^{j \gamma_{i}(n) \theta_{i}}\right] g^{-1}$, which takes off at $t\left(n_{0}\right)=I$ and is constant $t\left(n_{f}\right)=t$ from $n=n_{f}$ on. Similarly, for the reconstruction filter, the input matrix starts off from $s\left(n_{0}\right)=I$ and spirals along the trajectory $s(n)=g\left[\operatorname{diag}\left(q_{i}(n)\left|\lambda_{i}^{-1}\right|+\right.\right.$ $\left.\left.\left(1-q_{i}(n)\right)\right) e^{j-\gamma_{i}(n) \theta_{i}}\right] g^{-1}$ to end at constant $s\left(n_{f}\right)=s$ at time instant $n=n_{f}$. As explained in Proposition 2, perfect reconstruction is guaranteed whenever $q_{i}(n)=\frac{p_{i}(n)\left|\lambda_{i}\right|}{p_{i}(n)\left|\lambda_{i}\right|+\left(1-p_{i}(n)\right)}$.

The smooth transitions of both the spectral characteristics at the decomposition side and the impulse responses at the reconstruction side are clearly seen in the plots shown in Figs. 4 and 5, respectively, for channel four. The other channels transit in a likewise gentle way. As a final plot, the trajectories of the eigenvalues of $t(n)$ and $s(n)$ are shown in Fig. 6.

\section{REFERENCES}

[1] R. A. Gopinath and C. S. Burrus, "Factorization approach to unitary time-varying filter bank trees and wavelets," IEEE Trans. Signal Processing, vol. 43, pp. 666-680, Mar. 1995.

[2] C. Herley and M. Vetterli, "Orthogonal time-varying filter banks and wavelet packets," IEEE Trans. Signal Processing, vol. 42, no. 10, pp. 2650-2663, Oct. 1994.

[3] R. L. de Queiroz and K. R. Rao, "Time-varying lapped transforms and wavelet packets," IEEE Trans. Signal Processing, vol. 41, pp. 3293-3305, Dec. 1993.

14] K. Nayebi, I. Sodagar and T. P. Barnwell, "Time-varying filter banks and wavelets," IEEE Trans. Signal Processing, vol. 42, pp. 2983-2996, Nov. 1994.

[5] H. G. J. Theunis and E. F. Deprettere, "Piecewise stationary perfect reconstruction filter banks," AEU, Special Issue in Memory of W. Cauer, 1995.

[6] P. P. Vaidyanathan, Multirate Systems and Filter Banks. Englewood Cliffs, NJ: Prentice-Hall, 1993. 